\title{
Functional electrical stimulation for home-based upper-limb stroke rehabilitation
}

DOI 10.1515/cdbme-2017-0006

\begin{abstract}
Functional electrical stimulation (FES) therapies have shown effectiveness in restoring movement poststroke, especially when applied functionally to assist participants' voluntary intention during repeated, motivating tasks. Recent development in non-contact sensors allows feedback to advanced controllers that precisely adjust FES via an electrode array to assist functional reach and grasp tasks. This has given rise to significant reduction in impairment, as measured in clinical trials. This paper describes the recent developments of a compact system suitable for transference to patients' homes, with the intention of reducing upper-limb impairment following chronic stroke.
\end{abstract}

Keywords: functional electrical stimulation; iterative learning control; electrode-array technology; stroke rehabilitation.

\section{Introduction}

Stroke is the principal cause of adult disability in the UK, with an annual incidence of 152,000 people and a cost of $£ 9$ billion [1]. $77 \%$ of the 1.2 million stroke survivors experience altered arm function, and $40 \%$ are left with a nonfunctional arm [2]. This has a direct effect on independence, increasing the social and financial burden of stroke [3] There is strong evidence for physical therapy interventions which include intensive, highly repetitive task-oriented and task-specific training in all phases post stroke $[4,5]$. This presents a major challenge to healthcare providers and is driving the development of rehabilitation technology which can deliver this specific and intense rehabilitation without using additional resources.

Robotic systems have the ability to provide longer, more intense and more precise therapy. According to the functional independence measurement (FIM) index [6], robotic therapy at present provides no benefit over intensively matched conventional therapy [7]. A limitation of robotic therapy is its size and cost, which means it is not suitable for home-transference to provide intensive therapy. An alternative method of therapy is functional electrical stimulation (FES) which can provide intensive and effective rehabilitation in a home environment. It enables patients to practice meaningful, functional tasks and has been shown to improve range of movement, strength, and spasticity, while additionally having a positive effect on motor control [8]. Statistical evidence shows that benefits of FES are greatest when combined with maximum voluntary effort from the patient [9]. However, most upper-extremity (UE) rehabilitation systems that have been clinically trialled employ open-loop or triggered control of one or two muscles [10]. Iterative learning control (ILC) is an advanced control method that supports voluntary intention by enabling precise tracking task of predefined trajectories. It is one of the only model-based control approaches to have been used in clinical trials, and operates using data from previous attempts at the task to update the control input on the current execution. Previous studies combining FES and ILC have demonstrated feasibility of integrating an electrode array and single pad electrodes to deliver precisely controlled stimulation to wrist extensors, anterior deltoid and triceps $[11,12]$. However, their size and cost mean they are not suitable for home transference.

The system developed in this paper supports training of goal-oriented tasks through substantial innovations in the stimulation hardware, sensing equipment, control algorithms, and task display. In particular, the system integrates two important components. The first is a wireless unit combining an array of FES electrodes, and a microcontroller running the control algorithm. The second is a user terminal combining a laptop, graphical user interface (GUI), software and a single sensor unit (Kinect v2) that provides upper-limb joint positions in real-time.

\section{System overview}

Computer Science, University of Southampton, UK,

E-mail: mck1e12@ecs.soton.ac.uk

Chris Freeman and Matthew Spraggs: Electronics and Computer

Science, University of Southampton, UK
The UE rehabilitation system is named GO-SAIL (goaloriented stimulation assistance through iterative learning) 


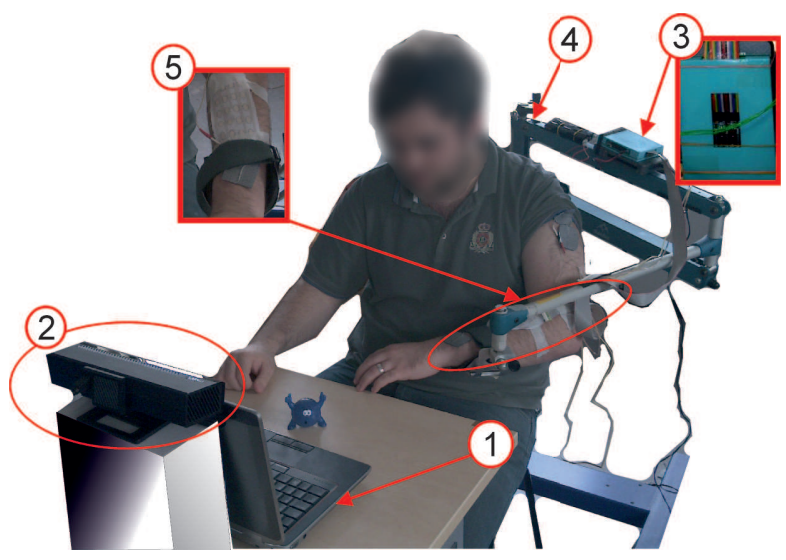

Figure 1: GO-SAIL Compact system components: (1) Laptop/tablet PC, (2) single sensor unit, (3) compact real time hardware and combined stimulator electronics, (4) arm support, (5) fabric electrode array.

Compact and is shown in Figure 1. An anti-gravity arm support is used to de-weight the participant's upper extremity according to individual need. The system trains functional task sets, comprising both real and virtual movements. The anterior deltoid and triceps are stimulated with surface electrodes and the common extensor complex of the forearm is stimulated with an electrode array. The GUI runs on the tablet PC/laptop and is employed to assign parameters and visualise results. It hence can be used by the carer, physiotherapist, or patient (via the unimpaired arm) to select tasks and monitor training progression. The Kinect v2 is used to measure the position of joint centres and is placed in front of the participant. The combined array stimulator electronics and compact real-time hardware are attached to the passive support as seen in Figure 1. In the future versions it is intended that this hardware may be integrated in the users' own clothing to maximise convenience. The system components are now described.

\subsection{Biomechanical model}

The reach and grasp tasks consist of repeated movements for the participant's affected arm, with a rest period in between during which their arm is returned to a common starting position. The requirement to repeatedly perform a set of finite duration tasks enables ILC to be utilised to control the FES signals for each muscle group. Each ILC trial starts from a fixed initial arm position and the performance error from each trial is used to update the control parameters in an attempt to increase the accuracy of the subsequent attempt. The control signal is generated using kinematic joint information, in combination with a

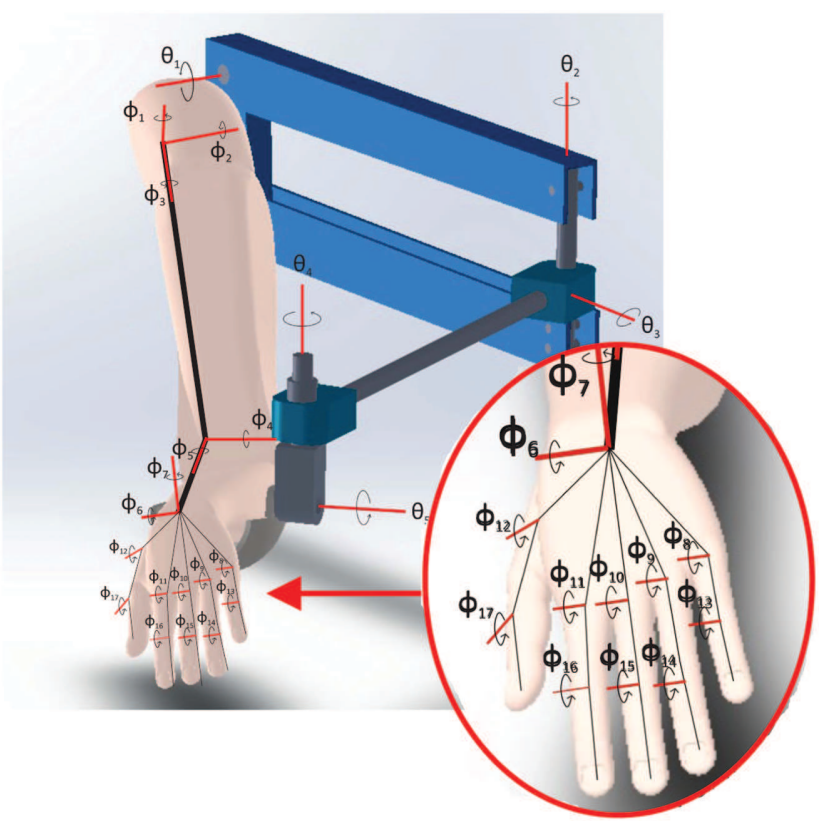

Figure 2: Human arm kinematic model with joint angles.

dynamic model of system described in [11], and given by

$$
\begin{gathered}
B(\Phi) \ddot{\Phi}+C(\Phi, \dot{\Phi}) \dot{\Phi}+F(\Phi, \dot{\Phi})+G(\Phi)+K(\Phi) \\
=\tilde{g}(u, \Phi, \dot{\Phi})-J^{\top}(\Phi) h
\end{gathered}
$$

where $B(\cdot)$ and $C(\cdot)$ are 17-by-17 inertial and Coriolis matrices respectively, $K(\cdot), F(\cdot)$ and $G(\cdot)$ are spring support, friction and gravitational vectors respectively, $h$ is a vector of external force and torque comprising components and $J(\cdot)$ is the system Jacobian. The vectors $\Phi=\left[\phi_{1}, \ldots, \phi_{17}\right]^{T}$ and $u=\left[u_{1}, \cdots, u_{26}\right]^{T}$ respectively denote joint angles and applied electrical stimulation. The vector $\tilde{g}(\cdot)$ comprises the moments produced through application of FES.

This model is next used by the FES control system to produce an input signal that results in accurate completion of each task.

\subsubsection{Control scheme}

In GO-SAIL Compact, $u_{a}=\left[u_{1}, u_{2}\right]^{\top}$ is the stimulation applied to the shoulder and elbow, and $u_{w}=\left[u_{3}, \cdots\right.$, $\left.u_{26}\right]^{\top}$ is the stimulation applied to the forearm muscles via the electrode array. In addition, $\Phi_{a}=\left[\phi_{1}, \cdots, \phi_{5}\right]^{\top}$ and $\Phi_{w}=\left[\phi_{6}, \cdots, \phi_{17}\right]^{\top}$ contain the joint angles of the upper arm and wrist respectively. The control scheme is shown in Figure 5 , in which the feedback controller is partitioned as $C_{c}=\operatorname{diag}\left\{C_{c, a}, C_{c, w}\right\}$ and is designed to establish stability and baseline tracking during each trial. The requirement to repeatedly perform a set of finite duration tasks with 
a fixed initial arm position enables ILC to be utilised to improve tracking performance. ILC uses the performance error from each trial to update the input $v_{k}=\left[v_{a, k}^{\top}, v_{w, k}^{\top}\right]^{\top}$ in an attempt to increase the accuracy of the subsequent attempt. On trial $k, \Phi_{k}(t)$ denotes the joint angles and the associated error is given by $e_{k}=\hat{\Phi}-\Phi_{k}$. Feedback controller component, $C_{c, a}$, is chosen to be an input-output linearising controller, in series with feedback controller, $K_{a}(s)=\left[0, K_{a, 2}(s), 0, K_{a, 4}(s), 0\right]^{\top}$ is then selected to stabilise the resultant closed-loop dynamics

$$
\begin{array}{r}
G_{a, i}:\left(\hat{\phi}_{i}+v_{k, i}\right) \mapsto \phi_{k, i}: \phi_{k, i}(s)= \\
\left(I+H_{a}(s) K_{a, i}(s)\right)^{-1} H_{a}(s) K_{a, i}(s)\left(\hat{\phi}_{i}(s)+v_{k, i}(s)\right), \\
i=2,4 .
\end{array}
$$

Note that $\phi_{2}$ and $\phi_{4}$ denote the anterior deltoid and elbow joint angles respectively.

A linearised model of the wrist $H_{w}(s): u_{w, k} \mapsto \Phi_{w, k}$ is identified about a suitable operating point. Feedback controller $C_{c, w}=K_{w}(s)$ is then chosen to stabilise system $H_{w}(s)$ to yield the resulting closed-loop dynamics

$$
\begin{aligned}
& G_{w}:\left(\hat{\Phi}_{w}+v_{w, k}\right) \mapsto \Phi_{w}: \Phi_{w}(s)= \\
& \left(I+H_{w}(s) K_{w}(s)\right)^{-1} H_{w}(s) K_{w}(s)\left(\hat{\Phi}_{w}(s)+v_{w, k}(s)\right) .
\end{aligned}
$$

An ILC scheme is then implemented in order to provide input $v_{k}$ such that the error is minimised, i.e. $\lim _{k \rightarrow \infty} v_{k}=v_{k}^{\star}$ with $v_{k}^{\star}:=\min _{v_{k}}\left\|\hat{\Phi}-\Phi_{k}\right\|^{2}$. This is achieved through the update structure

$$
v_{k+1}=v_{k}+L e_{k}, \quad v_{0}=0, \quad k=0,1, \cdots
$$

where $L=\operatorname{diag}\left\{L_{a}, L_{w}\right\}$.

For the arm dynamics, design of $L_{a, i}$ to satisfy $\| I-$ $G_{a, i} L_{a, i} \|<1, i=2$, 4, guarantees convergence of $\phi_{i}$ to zero error, and many suitable schemes are available, see [13] and examples therein. Similarity, $\left\|I-G_{w} L_{w}\right\|<1$ guarantees convergence of the wrist and hand joints to zero error. If this is not possible, the alternative form of $\| I-$ $G_{w} L_{w} \|<1$ may be employed to guarantee convergence of error $e_{w, k}$ to the limiting solution $\left(I-G_{w}\left(L_{w} G_{w}\right)^{-1} L_{w}\right) \hat{\Phi}_{w}$.

\section{Experimental results}

Feasibility was established in preliminary tests with unimpaired participants who provided no voluntary effort. The set-up procedure included placing of electrodes, setting

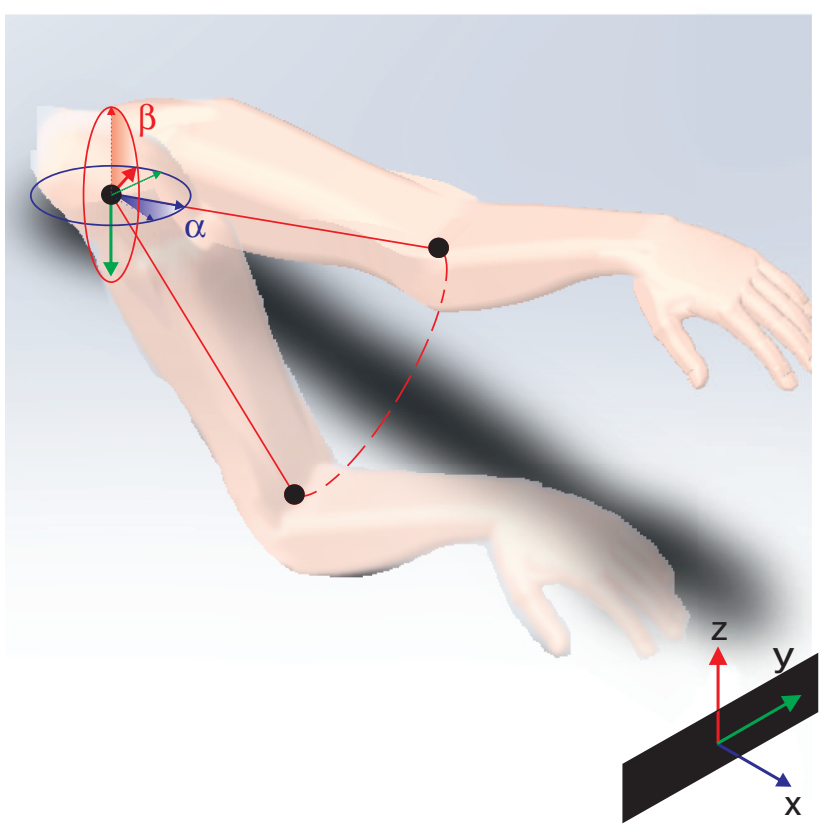

Figure 3: Anterior deltoid identification with parameters $\alpha, \beta$.

amplitudes and performing identification tests to find the model components $\alpha, \beta$ and the static $P$ matrix which relates the array stimulation with the corresponding movement of the hand and wrist. Here $\alpha$ and $\beta$ are parameters introduced into the kinematic chain in order to align the axis corresponding to joint angle $\phi_{2}$ with the movement produced by stimulating the anterior deltoid. Figure 3 illustrates these parameters.

Identification of the upper arm components involves identifying the stimulated joint axes within the kinematic model [14]. This step is achieved by applying a ramped $10 \mathrm{~s}$ FES signal to the anterior deltoid and fitting a plane to the resulting movement. Transformations are then embedded in the kinematic chain to align the $\phi_{2}$ axis to the plane normal. Numerous methods can be used to identify the hand and wrist dynamics around an operating point which corresponds to the kth trial, including fitting a linear model to the trial data set or selecting a model from a set identified over a range of operating points at the beginning of the treatment session. Here the former approach in [15] was used to yield $P$.

After completing the set-up procedure, the participant opens the application with his/her unimpaired arm and starts a selection of tasks. Each task was repeated a total of six times.

Test results confirmed high levels of performance over a range of functional tasks. Initial results from hand and wrist tracking are shown in Figure 4. Further feasibility study is underway and will be reported separately. 

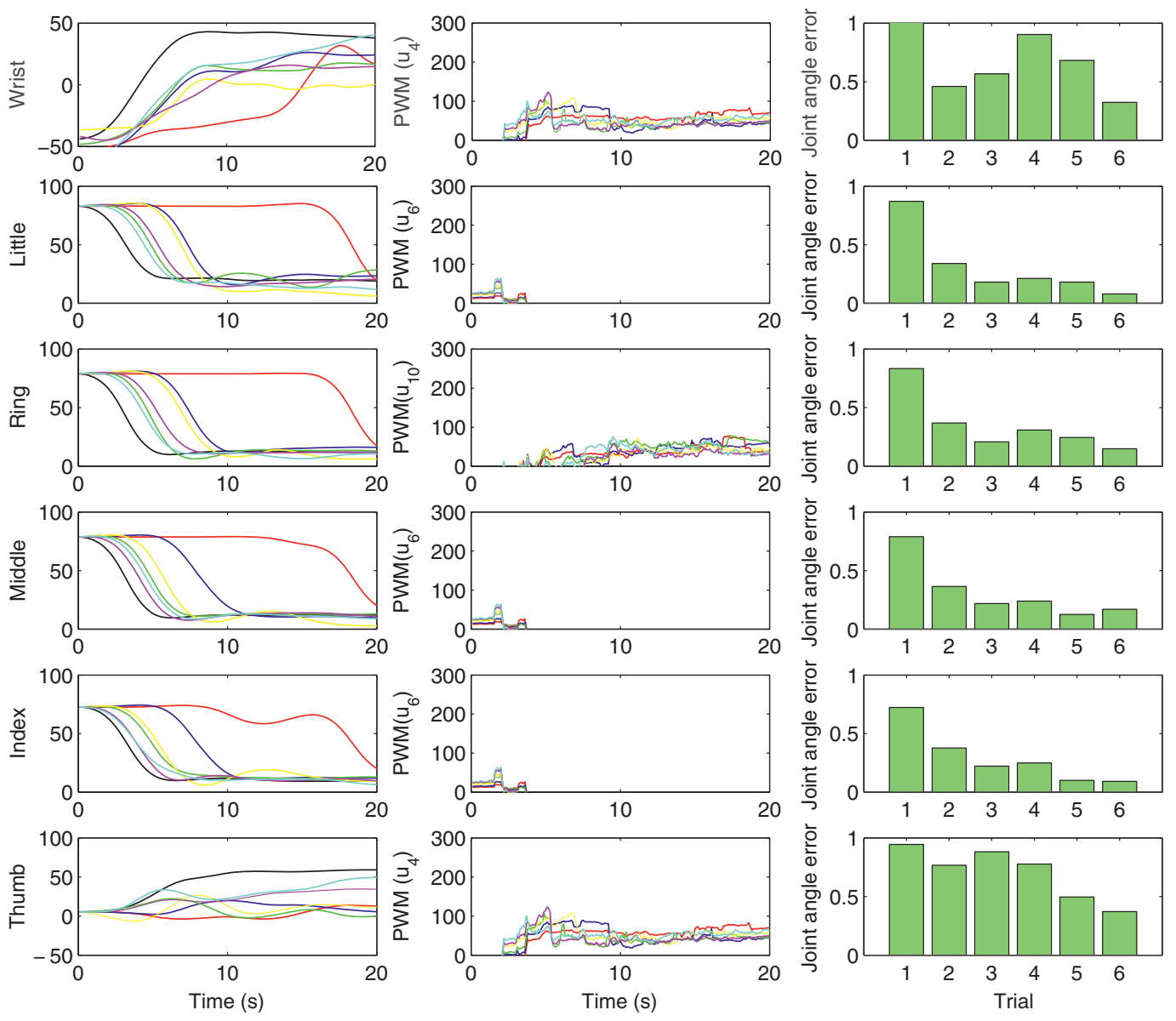

$-5-6$

Figure 4: Participant 1's hand and wrist data for open hand movement.

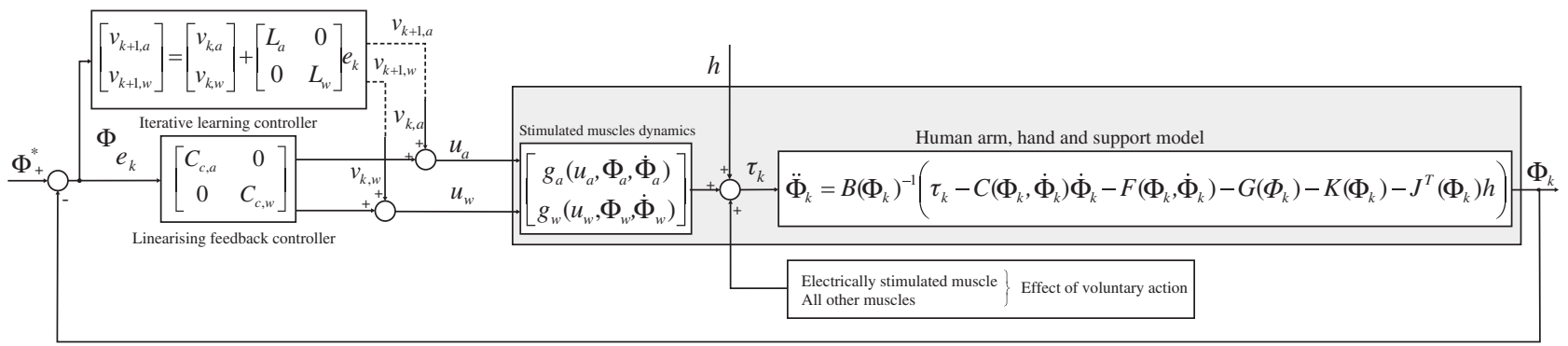

Figure 5: Block diagram of the ILC feedback scheme. Note that the dashed line denotes computer memory updating of ILC signal.

\section{Conclusion}

The aim of this paper was to establish the design of a compact system combining state-of-the-art technologies to enable people with stroke to practise goal-oriented functional tasks at their home. It incorporates VR, FES hardware, advanced sensing, control and passive support. Preliminary results confirmed that FES, mediated by ILC, successfully assisted participants' completion of functional tasks and training transferred to tangible changes in motor performance. Finally, the feasibility of using low-cost, userfriendly sensing approaches and arm support mechanisms that can be used in conjunction with FES-assisted tasks was established and comprise an important step towards the transference of such a rehabilitation system to the home. What remains unclear however is the usability of the system for stroke participants. Thus, a feasibility study will be performed to explore usability of the system in the home environment. 


\section{Author's Statement}

Research funding: This work was supported by the Engineering and Physical Sciences Research Council (EPSRC) Grant no. EP/M026388/1. Conflict of interest: Authors state no conflict of interest. Informed consent: Informed consent is not applicable. Ethical approval: The conducted research is not related to either human or animal use.

\section{References}

[1] Townsend N, Wickramasinghe K, Bhatnagar P, Smolina K, Nichols $M$, Leal J, et al. Coronary heart disease statistics. Technical report, British Heart Foundation; 2012.

[2] Intercollegiate stroke working party. National clinical guideline for stroke. Technical report, London: Royal College of Physicians; 2012.

[3] Kwakkel G. Intensity of practice after stroke: more is better. Power 2009;7:24.

[4] Timmermans A, Seelen H, Willmann R, Bakx W, De Ruyter B, Lanfermann G, et al. Arm and hand skills: training preferences after stroke. Disabil Rehabil. 2009;31:1344-52.

[5] Winstein C, Stein J, Arena R, Bates B, Cherney LR, Cramer $S C$, et al. Guidelines for adult stroke rehabilitation and recovery a guideline for healthcare professionals from the american heart association/american stroke association. Stroke 2016;47:e98-169.

[6] Van der Loos M. Robot-assisted movement training compared with conventional therapy techniques for the rehabilitation of upper-limb motor function after stroke. Arch Phys Med Rehabil. 2002;83:952-9.

[7] Mehrholz J, Pohl M, Platz T, Kugler J, Elsner B. Electromechanical and robot-assisted arm training for improving activities of daily living, arm function, and arm muscle strength after stroke. Cochrane Library; 2015.

[8] Urton M, Kohia M, Davis J, Neill M. Systematic literature review of treatment interventions for upper extremity hemiparesis following stroke. Occup Therapy Int. 20007;14:11-27.

[9] Rushton D. Functional electrical stimulation and rehabilitation an hypothesis. Med Eng Phys. 2003;25:75-8.

[10] Pelton T, van Vliet P, Hollands K. Interventions for improving coordination of reach to grasp following stroke: a systematic review. Int J Evid-Based Healthcare. 2012;10:89-102.

[11] Kutlu M, Freeman CT, Hallewell E, Hughes AM, Laila DS. Upper-limb stroke rehabilitation using electrode-array based functional electrical stimulation with sensing and control innovations. Med Eng Phys. 2016;38:366-79.

[12] Meadmore KL, Exell T, Hallewell E, Hughes AM, Freeman $\mathrm{CT}$, Kutlu M, et al. The application of precisely controlled functional electrical stimulation to the shoulder, elbow and wrist for upper limb stroke rehabilitation: a feasibility study. J NeuroEng Rehabil. 2014;11:105.

[13] Freeman CT. Electrode array-based electrical stimulation using ILC with restricted input subspace. Control Eng Pract. 2014;23:32-43.

[14] Freeman CT. Constrained point-to-point iterative learning control with experimental verification. Control Eng Pract. 2012;20:489-98.

[15] Freeman CT, Yang K, Tudor J, Kutlu M. Feedback control of electrical stimulation electrode arrays. Med Eng Phys. 2016;38:1185-94. 